\title{
Morbilidad materna grave e ingreso a cuidado intensivo. Hospital Clínico Universidad de Chile (2006-2010)
}

\author{
JORGE HASBÚN H. ${ }^{1}$, ALVARO SEPÚLVEDA-MARTÍNEZ ${ }^{1}$, \\ RODRIGO CORNEJO R. ${ }^{2}$, CARLOS ROMERO P. ${ }^{2}$
}

${ }^{1}$ Unidad de Medicina Materno-Fetal, Hospital Clínico Universidad de Chile. Santiago de Chile. ${ }^{2}$ Unidad de Pacientes Críticos, Hospital Clínico Universidad de Chile. Santiago de Chile.

Recibido el 1 de enero de 2013, aceptado el 21 de octubre de 2013.

Correspondencia a: Dr. Jorge Hasbún $\mathrm{H}$. Santos Dumont 999 sector A primer Piso, Independencia. Santiago de Chile.

Fono: 56(2)9788881 E-mail: ecosonica@gmail.

\section{Intensive care admissions due to severe maternal morbidity}

Background: Maternal morbidity is a quality of care indicator. The frequency of severe maternal morbidity that requires an intensive care management has increased, due to an increase in maternal age. Aim: To describe the severe and acute maternal morbidity spectrum that requires an intensive care management in a University Hospital. Material and Methods: Review of medical records of 89 pregnant women with a mean age of 29 years, admitted to an Intensive Care Unit (UCI) between 2006 and 2010. Results: Mean gestational age on admission was 32 weeks. The main comorbidities identified were chronic hypertension (13.5\%), hypothyroidism (4.5\%) and coagulopathies (6.7\%). Severe preeclampsia, sepsis and obstetric hemorrhage were the main causes of admission. Length of stay ranged from 1 to 28 days. Seventy eight percent of patients were admitted in the immediate postnatal period. Mechanical ventilation was required in $24 \%$ of patients for a median of three days. The longer unit lengths of stay were observed in patients with preeclampsia and non-obstetric severe sepsis (pyelonephritis and pneumonia). Seven abortions and seven perinatal deaths were recorded. The latter were mainly secondary to severe preeclampsial HELLP syndrome. Neonatal morbidity was related to prematurity (19\% hyaline membrane, $18 \%$ persistent ductus and $4 \%$ cerebral hemorrhage). There were no maternal deaths. Conclusions: Preeclampsia and its complications were the main causes of maternal ICU admission. In this series, there were no maternal deaths and the perinatal survival rate was $92 \%$.

(Rev Med Chile 2013; 141: 1512-1519)

Key words: Intensive care; Maternal mortality; Perinatal mortality; Preeclampsia; Sepsis.
L a mortalidad materna es un indicador tradicional de calidad de atención médica para un período determinado, pero actualmente, en países desarrollados, la muerte materna es infrecuente y su tendencia temporal es estable ${ }^{1}$. Su sensibilidad como marcador de calidad es insuficiente y su análisis aislado induce a subvalorar otros problemas importantes del cuidado médico obstétrico.

La morbilidad materna puede alcanzar a $43 \%$ de las pacientes ${ }^{2}$ y es considerada un espectro continuo de riesgo, con etapas progresivas desde la embarazada sana expuesta a un insulto, a morbilidad leve, moderada, grave aguda, grave con cercanía a la muerte ("near miss") y la muerte materna ${ }^{3}$.

Este concepto identifica mejor las causas y factores determinantes de la evolución de una etapa a otra susceptible de intervenir y pudiendo optimizar el indicador en países desarrollados con baja incidencia de muerte materna ${ }^{4}$. 
En Chile, la mortalidad materna descendió a 22 por 100.000 nacidos vivos en dos décadas, pero luego se ha mantenido estable ${ }^{5,6}$ y se ha planteado que para los países en desarrollo la morbilidad puede ser un indicador útil y su estudio puede contribuir en la disminución de la mortalidad materna $^{7,8}$.

Las embarazadas que ingresan a Unidad de Cuidados Intensivos (UCI), en su mayoría son casos agudos en condición crítica y riesgo de muerte, que necesitan tratamiento especializado y son un grupo significativo de la práctica obstétrica9.

El objetivo del estudio es describir el espectro de la morbilidad materna grave y aguda que requiere este nivel de manejo médico.

\section{Material y Métodos}

En estudio retrospectivo de serie de casos se revisan los registros clínicos de embarazadas trasladadas desde la Unidad de Medicina MaternoFetal de Maternidad a la UCI del Hospital Clínico de la Universidad de Chile en el período enero de 2006 a diciembre de 2010.

Ambas unidades están en un Centro ClínicoAcadémico terciario y de referencia que atiende una población homogénea de clase media que es asistida por obstetras e intensivistas, ambos disponibles las $24 \mathrm{~h}$ del día, en interacción continua para las decisiones médicas.

Se utilizó como instrumento una ficha diseñada con datos demográficos, diagnóstico primario (causa del ingreso), momento de admisión, complicaciones, intervenciones, permanencia intrahospitalaria y factores de riesgo. Se evaluó el pronóstico materno y el pronóstico perinatal (mortalidad y morbilidad).

\section{Análisis estadístico}

Para las variables continuas se realiza prueba de Kolmogorov-Smirnov para determinación de normalidad. En caso de distribución normal, los datos se expresan en media y desviación estándar; en distribución no paramétrica los datos se expresan en mediana y rango.

Las variables categóricas son dicotomizadas y expresadas en porcentajes. Todos los análisis fueron desarrollados con el programa estadístico SPSS v 15.0 .

\section{Resultados}

Durante el período 2006-2010 hubo 89 ingresos a UCI con incidencia de $4,1 \%$ del total de egresos de Maternidad $(\mathrm{n}=2.169)$ y $1,18 \%$ del total de partos $(\mathrm{n}=7.513)$.

La data demográfica (Tabla 1) demuestró que eran pacientes jóvenes (promedio 29,4 años), que se complicaron predominantemente en el tercer trimestre (mediana de 32 semanas) y tuvieron distribución similar según paridad.

En la Tabla 2 se observa el diagnóstico de ingreso a UCI y su frecuencia relativa, siendo hipertensión arterial, infección y hemorragia obstétrica las causas principales. La preeclampsia representó $47,2 \%$ de los casos ( 42 /89), que incluyó 23 pacientes con síndrome HELLP, 3 con eclampsia y casos con un espectro amplio de complicaciones agudas: hemorragia, distress respiratorio, coagulación intravascular diseminada e insuficiencia renal.

\section{Tabla 1. Características demográficas de embarazadas UCI $(n=89)$}

\begin{tabular}{|lc|}
\hline Edad (promedio $\pm \mathrm{DE})$ & $29,4 \pm 7,1$ \\
\hline Edad gestacional (mediana, rango) & $32(9-41)$ \\
\hline Primigesta ( $\mathrm{n} \%$ del total) & $42(47,2)$ \\
Multípara & $47(52,8)$ \\
\hline
\end{tabular}

$\mathrm{DE}=$ desviación estándar.

Tabla 2. Diagnóstico de ingreso embarazadas UCI ( $n=89$ )

\begin{tabular}{|lcc|}
\hline Patología & $\begin{array}{c}\text { n de } \\
\text { casos }\end{array}$ & $\%$ \\
\hline Preeclampsia grave & 42 & 47,2 \\
\hline $\begin{array}{l}\text { Infecciones } \\
\text { Infecciones no obstétricas }\end{array}$ & 24 & 26,9 \\
$\quad$ Infecciones obstétricas & 7 & 19,1 \\
Hemorragia obstétrica & 16 & 17,8 \\
Complicación aguda hemodinámica o & 5 & 5,6 \\
neurológica & 3 & 3,4 \\
Cardiopatía grave & 3 & 3,4 \\
\hline Enfermedades de tejido conectivo & 3 & 3,4 \\
\hline latrogénicas & 1 & 1,1 \\
\hline Hígado graso agudo & & \\
\hline
\end{tabular}


Morbilidad materna grave en UCl - J. Hasbún et al

Infección fue la segunda causa de ingreso con 24 casos $(26,9 \%), 13$ de los cuales fueron pielonefritis, principal causa antenatal $(14,6 \%$ del total). Hubo 2 neumonías, 2 infecciones virales, 3 corioamnionitis, 4 abortos sépticos y 5 pacientes requirieron ventilación mecánica (VM) confirmando el riesgo de distress respiratorio del adulto en la paciente séptica y la importancia de la ventilación protectora del pulmón.

Hubo 16 pacientes con hemorragia obstétrica (18\%): 9 casos de inercia uterina, 6 casos de percretismo placentario y 1 caso de hematoma placentario con tromboembolismo.

En inercia uterina el volumen promedio transfundido (coloides más cristaloides) fue de $3.385 \mathrm{ml}$ (más un promedio de 1,1 unidades de glóbulos rojos) y la estadía en UCI fue 3,8 días. Tres pacientes tuvieron pre-eclampsia grave y hubo casos únicos de edema pulmonar agudo, insuficiencia renal y distress respiratorio.

Hubo 6 casos de acretismo placentario: 5

Tabla 3. Estadía y evolución en UCI $(n=89)$

\begin{tabular}{|lc|}
\hline Días de estadía (mediana, rango) & $3(1-28)$ \\
\hline Ventilación mecánica $(n, \%)$ & $21(23,6)$ \\
$\begin{array}{l}\text { Días en ventilación mecánica (promedio, } \\
\text { rango) }\end{array}$ & $3,7(1-22)$ \\
\hline $\begin{array}{l}\text { Egresos } \mathrm{UCI}(\mathrm{n}, \%) \\
\text { Muerte materna }(\mathrm{n}, \%)\end{array}$ & $89(100)$ \\
\hline
\end{tabular}

tuvieron cesárea-histerectomía en interrupción electiva, con edad gestacional promedio de 35,2 semanas (mediana 35; rango 34-38), obtenida la mayor madurez fetal y anticipando una hemorragia cataclítmica propia. Un caso urgente correspondió a un percretismo perforante espontáneo y rotura uterina en semana 18 del embarazo. El volumen transfundido en acretas antes del traslado a UCI fue $6.916 \mathrm{ml}$ (coloides + cristaloides) y 4,8 unidades de glóbulos rojos (promedios).

Otras causas menos frecuentes de ingreso fueron complicación aguda hemodinámica o neurológica (edema agudo de pulmón y estatus convulsivo en paciente epiléptica), cardiopatía grave, enfermedades del tejido conectivo (lupus, SAAF), iatrogénicas (EPA por uso de betamiméticos, shock anafiláctico por anestesia) e hígado graso agudo.

En las Tablas 3 y 4 se observa la evolución de las pacientesen UCI y los días en ventilación mecánica.

La mediana de estadía en UCI para el total de pacientes fue de 3 días. En 22 casos $(24,7 \%)$ la permanencia fue el doble o más de la mediana (más de 5 días), sugiriendo respuesta más lenta a la terapia.

La evolución en UCI según diagnóstico demuestra que la infección no obstétrica fue la enfermedad con promedio de estadía más prolongada: 13 pacientes con pielonefritis ( 6,1 días), 2 pacientes con neumonía (5,5 días), 2 pacientes con sepsis de origen intestinal (1 día).

Tabla 4. Evolución en UCI según patología de ingreso* $(n=89)$

\begin{tabular}{|lccc|}
\hline Patología & EG al ingreso hospitalario & Días en UCI & Días en VM \\
\hline PES / HELLP / Eclampsia & $32(21-40)$ & $3(1-19)$ & $0(0-11)$ \\
\hline Acretismo placentario & $35(19-36)$ & $3(1-7)$ & $0(0-2)$ \\
\hline Cardiopatía grave & $33(33-$ puerp) & $2(2-7)$ & $0(0)$ \\
\hline Infección obstétrica & $18(14-31)$ & $1(1-4)$ & $0(0)$ \\
Infección no obstétrica & $28(14-37)$ & $5(1-18)$ & $0(0-11)$ \\
\hline Status convulsivo & $33,5(29-36)$ & $3,5(1-17)$ & $0(0)$ \\
\hline Edema pulmonar aguda & $34(34-$ puerp) & $2,5(2-3)$ & $0,5(0-1)$ \\
\hline Perforación intestinal** & puerp & 28 & 22 \\
\hline
\end{tabular}

$\mathrm{PES}=$ Pre-eclampsia severa; $\mathrm{UCl}=$ Unidad de Cuidados Intensivos; $\mathrm{VM}=$ ventilación mecánica; $\mathrm{EG}=$ Edad gestacional; puerp $=$ ocurrencia en puerperio. ${ }^{*}$ Datos expresados en mediana y rango. ${ }^{* *}$ un caso. 
Morbilidad materna grave en UCI - J. Hasbún et al

Tabla 5. Resultado perinatal en recién nacidos de egresadas $\mathrm{UCI}$

\begin{tabular}{|c|c|}
\hline EG al parto (feto vivo)* & $34(26-41)$ \\
\hline $\begin{array}{l}\text { Peso de nacimiento (feto vivo) } \\
\text { gramos* }\end{array}$ & $2.082(730-4.100)$ \\
\hline APGAR $<7 \mathrm{al} \mathrm{mi}^{\dagger}$ & $20(22,5)$ \\
\hline APGAR $<7$ a los $5 \mathrm{~min}^{\dagger}$ & $5(5,6)$ \\
\hline Muerte antenatal $^{\dagger}$ & $4(4,3)$ \\
\hline Muerte neonatal ${ }^{\dagger}$ & $3(3,4)$ \\
\hline $\begin{array}{l}\text { Mortalidad perinatal } \\
\text { (x } 1.000 \text { embarazadas UCI) }\end{array}$ & 78,6 \\
\hline Secuela al alta ${ }^{\dagger}$ & $13(18,6)$ \\
\hline
\end{tabular}

*datos en mediana (rango); 'datos en número de casos (porcentaje); $\mathrm{n}=92$ ( 3 embarazos múltiples). No se incluyen 5 abortos sépticos $<21$ semanas. EG $=$ edad gestacional.

Tabla 7. Antecedentes mórbidos de ingresadas $\mathrm{UCI}(\mathrm{n}=\mathbf{8 9})$

\begin{tabular}{|lcc|}
\hline Antecedentes & n de casos & \% \\
\hline Hipertensión arterial crónica & 12 & 13,5 \\
Asma & 3 & 3,4 \\
SAAF & 2 & 2,2 \\
Lupus & 1 & 1,1 \\
Coagulopatías & 6 & 6,7 \\
Trombofilia & 1 & 1,1 \\
TVP previa & 4 & 4,5 \\
Trombocitopenia & 3 & 3,4 \\
Endocrinopatías & 8 & 9 \\
Hipertiroidismo & 1 & 1,1 \\
Hipotiroidismo & 4 & 4,5 \\
Enfermedad de Cushing & 1 & 1,1 \\
Diabetes Mellitus 1 & 1 & 1,1 \\
\hline Diabetes Mellitus 2 & 0 & 0 \\
Hiperandrogenismo & 1 & 1,1 \\
\hline
\end{tabular}

SAAF = Síndrome anticuerpos antifosfolípidos; TVP = Trombosis venosa profunda.

Las mayores estadías correspondieron a 1 caso de perforación intestinal ( 28 días), 1 caso de pielonefritis complicada con shock séptico (10 días) y 4 pacientes con pre-eclampsia también tuvieron estadía prolongada (10, 17, 18 y 19 días).

La VM fue utilizada en 21 casos $(23,6 \%), 7$ embarazadas y 14 puérperas. La duración de la
Tabla 6. Morbilidad neonatal RN $\mathbf{n}=\mathbf{8 9}$

\begin{tabular}{|lcc|}
\hline Evento neonatal & $\begin{array}{c}\text { n de } \\
\text { casos }\end{array}$ & \% \\
\hline Membrana hialina & 14 & 19,2 \\
\hline Ductus persistente & 13 & 17,8 \\
\hline Hemorragia intracerebral & 3 & 4,1 \\
Enterocolitis necrotizante & 0 & 0 \\
\hline Sepsis neonatal & 6 & 8,2 \\
\hline Displasia broncopulmonar & 1 & 1,4 \\
\hline Días en UCIN* & $4(0-57)$ & \\
\hline
\end{tabular}

$\mathrm{RN}=$ recién nacido. $\mathrm{UCIN}=$ unidad de cuidados intensivos neonatal. *Datos expresados en mediana (rango)

VM antenatal fue 2,8 días promedio (rango 1-9) y post-parto fue de 4,2 días (rango 1-22).

Las Tablas 5 y 6 muestran el resultado perinatal y las complicaciones neonatales destacando un alto número de partos prematuros y siete casos de muerte perinatal $(7,7 \%)$. La morbilidad respiratoria severa fue la patología más frecuente en los neonatos que requirieron manejo intensivo neonatal, con 19,2\% de los casos.

En la Tabla 7 se señalan los antecedentes mórbidos: hipertensión arterial crónica $(13,5 \%)$ e hipotiroidismo $(4,5 \%)$ son las patologías pregestacionales más frecuentes siendo ambas un factor de riesgo para pre-eclampsia, y luego coagulopatías e inmunológicas.

El antecedente de morbilidad obstétrica previa tuvo baja incidencia en nuestra casuística debido al alto número de primigestas jóvenes, sin embargo, cinco casos presentaron antecedente de cesárea anterior, factor mórbido determinante del acretismo placentario,causante del ingreso a UCI.

\section{Discusión}

Los problemas a resolver más importantes del manejo médico fueron la severidad del compromiso materno, la viabilidad y sobrevivencia del neonato, la continuación o la interrupción del embarazo, el modo o vía del parto y el efecto farmacológico de drogas. Estos problemas se dis- 
cutieron caso a caso entre obstetra e intensivista y las decisiones adoptadas fueron relevantes en el resultado.

En la discusión analizamos tres aspectos: 1) Causas del ingreso a UCI; 2) Importancia del parto y 3) Sobrevivencia perinatal.

\section{Causas de ingreso a UCI}

Las causas descritas de morbilidad materna grave que requieren cuidado intensivo son hemorragia 20 a $50 \%{ }^{10}$, hipertensión 10 a $45 \%^{11,12}$ e infección 8 a10\% ${ }^{13}$.

En esta serie, preeclampsia severa (PES) fue la primera causa de ingreso $(47,2 \%)$, similar a otros centros: $44,5 \%$ y $36,6 \%{ }^{14,15}$.

HELLP es una variante de preeclampsia severa definida por una tríada: $\mathrm{H}=$ hemólisis, $\mathrm{EL}=$ enzimas hepáticas elevadas, $\mathrm{LP}=$ trombocitopenia, que acumula los mayores riesgos de morbilidad grave, que afecta a $20 \%$ de las $\mathrm{PES}^{16}$, pero en esta serie su incidencia fue de $54,7 \%$.

Esto se debe al criterio diagnóstico que, además de la preeclampsia, incluyó la variedad parcial de HELLP, antes de su expresión completa,con uno o dos componentes de la tríada clásica $(H$, EL o LP). Posteriormente, al surgir en su evolución signos de deterioro materno (hipertensión refractaria, oliguria, compromiso neurológico, desprendimiento placentario) o deterioro fetal (signos de adaptación hemodinámica, asfixia, ausencia de reactividad), se efectuó interrupción más temprana, sin sus complicaciones evolutivas propias esperadas y más graves, como trombocitopenia irreversible, rotura hepática, CID, muerte materna, que no fueron observadas en esta serie.

También explican la mayor incidencia relativa la referencia de casos desde otros centros externos (aumenta el numerador) y la exclusión de PES tratadas en Maternidad con cuyo parto hubo remisión y mejoría sin ingreso a UCI (disminuye el denominador).

Otro factor es el uso universal de la técnica Doppler de arterias uterinas en el primer y segundo trimestre en las embarazadas controladas en la Unidad Materno-Fetal, como predictor de PES $^{17,18}$ que ha permitido la pesquisa temprana de pacientes susceptibles, con seguimiento y manejo oportunos.

Existe amplia evidencia que avala este procedimiento en un modelo predictivo de PES en la población general de embarazadas, con tasas de detección de $90 \%{ }^{19,20}$ y que ha permitido la intervención farmacológica preventiva en pacientes de alto riesgo ${ }^{21-24}$.

En estudio retrospectivo de cohorte con 442 pacientes complicadas con síndrome HELLP la mortalidad materna fue de $1,1 \%{ }^{16}$, pero ésta asciende en forma significativa en complicaciones como eclampsia, CID y rotura hepática ${ }^{30}$. En esta serie, la interrupción del embarazo antes de la aparición de estas complicaciones fue un factor importante en el resultado sin muertes maternas.

La permanencia breve en UCI de las pacientes con PES (mediana 3 días, rango 1-19) indica la evolución a mejoría rápida en respuesta a un diagnóstico y tratamiento adecuado y oportuno.

La infección es la primera causa de muerte materna directa en el Reino Unido ${ }^{25}$ y se asocia con sepsis materna y prematurez, requiere tratamiento antibiótico precoz y/o erradicación del foco ${ }^{26}$, objetivos logrados en esta serie.

La hemorragia representó 18\% de los ingresos, incidencia menor a lo descrito en otras series y que se explica por el manejo obstétrico adecuado en Maternidad con medidas anticipatorias en casos con factores de riesgo de inercia uterina, interrupción programada en casos potenciales de gran hemorragia como la placenta acreta y tratamiento precoz en hemorragias post-parto (Figura 1).

En acretismo, la reposición de volumen fue intraoperatoria en pabellón y el ingreso a UCI fue postparto por requerimiento mayor en el nivel de cuidado ya que en estas pacientes hubo

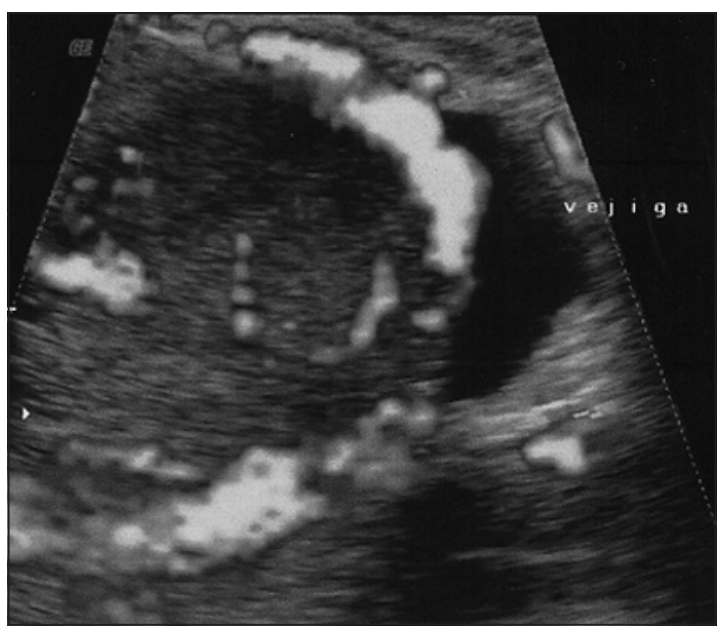

Figura 1. Ecografía Power Doppler de paciente portadora de placenta percreta con compromiso de mucosa vesical. 
invasión vascular intraoperatoria (monitoreo hemodinámico con catéter central, hemodilución hipervolémica o normovolémica, politranfusión, ligaduras o pinzamiento o embolización arteriales) y cirugía urológica complementaria.

El volumen promedio transfundido en acretismo en esta serie antes del traslado a UCI fue $6.916 \mathrm{ml}$ (coloides + cristaloides) y 4,8 unidades de glóbulos rojos, se compara favorablemente con el volumen transfundido reportado de $12.140 \pm$ $8.343 \mathrm{ml}^{27}$.

Pese a mayor hemorragia, reposición de volumen y cirugía más invasiva (5 cesárea-histerectomías y procedimientos complementarios) las pacientes con acretismo placentario permanecieron un período similar o menor en UCI y tuvieron menos complicaciones graves que las inercias uterinas. Este resultado fue favorecido por el uso planificado de nuevas técnicas quirúrgicas protectoras con mejor control del sangrado intraoperatorio ${ }^{28}$.

La importancia de la VM antenatal es que las drogas para sedación, analgesia y bloqueo neuromuscular tienen pasaje transplacentario y dificultan la evaluación fetal (por disminución de la variabilidad de la frecuencia cardiaca basal, pérdida de movimientos fetales, sueño fetal prolongado, perfil biofísico alterado); pueden ocultar una urgencia como desprendimiento placentario oun parto repentino en UCI o la necesidad eventual de una cesárea perimortem.

Iatrogenia: Hubo tres pacientes con iatrogenia por tratamiento médico: una insuficiencia cardiaca por betamiméticos, un shock anafiláctico secundario a anestesia y un caso de edema agudo de pulmón por sobrecarga en inercia uterina, resueltos con terapia específica.

Hubo dos pacientes con daño quirúrgico en acretas: una sutura vesical retráctil que evolucionó posteriormente con obstrucción ureteral y un caso de hemoperitoneo, ambas requirieron laparotomía. En percretismo vesical por definición hay invasión placentaria transmural de mucosa por lo que la cistostomía quirúrgicay la cistorrafia son de necesidad y no se consideran iatrogenias.

\section{Parto e ingreso a UCI}

En esta serie, 20 pacientes (22,4\%) ingresan en el período antenatal principalmente por pielonefritis del segundo trimestre y 69 casos $(77,6 \%)$ ingresan después del nacimiento. El ingreso fue determinado por la evolución de la patología de base, o bien, resuelto el embarazo, por la necesidad de cuidado intensivo post-parto.

Los partos fueron por indicación médica, decisión asumida en el momento estimado clínicamente de máximo riesgo materno y menor riesgo perinatal por prematurez, efectuandose el parto electivo antes del traslado a UCI.

No hubo partos inesperados en UCI ni traslados a maternidad para parto y luego retorno a UCI, complicaciones o inconvenientes de posible ocurrencia, pero que fueron evitadas por el manejo adecuado.

Pese a la condición continua de morbilidad grave progresiva, la sobrevivencia materna fue de $100 \%$, por lo que la interrupción y traslado a UCI en cada caso evitó una progresión fataly la decisión asumida fue oportuna. Este resultado se compara favorablemente con dos series de Sud-América ${ }^{15,29}$, que comunican una mortalidad materna de $11 \%$ y $33,8 \%$ respectivamente, pero que incluyen casos con deficiente cuidado prenatal y sepsis por aborto.

\section{Sobrevivencia perinatal}

La tasa de sobrevivencia fue de $92 \%$ ( 80 recién nacidos del total de 87 que incluye 3 gemelares).

Hubo 7 muertes fetales sin posibilidades de sobrevivencia ( 5 abortos y 2 prematuros extremos en semanas 20 y 24) y 7 muertes perinatales mayores a 24 semanas, todas en pacientes con PES y su variante HELLP.

La mortalidad perinatal reportada en PESHELLP es de 7,4 a $34 \%(31,32)$ y es mayor en los neonatos que nacen antes de las 32 semanas (33). En esta serie hubo 7 muertes en 23 casos (30,3\%) confirmando el impacto de esta patología materna sobre el pronóstico perinatal.

En este grupo, 3 casos ingresaron con el feto muerto in utero por acceso tardío de la paciente al mayor nivel de cuidado; 1 feto falleció durante la hospitalización por tardanza en la interrupción y en 3 casos la muerte ocurrió en el período neonatal por complicaciones de la prematurez.

En los casos de derivación tardía, no se alcanza a efectuar la profilaxis del distress respiratorio con la administración antenatal del corticoide y la prevención debe orientarse a un control antenatal más estrecho y una consideración más acotada de los factores de riesgo o antecedentes mórbidos que orienten a una hospitalización más oportuna. 
Morbilidad materna grave en UCI - J. Hasbún et al

Estos factores del cuidado médico y del sistema de atención deben ser revisados y optimizados en el manejo actual de la morbilidad para reducir la pérdida perinatal.

En conclusión, se identificaron las causas de morbilidad materna grave que requieren ingreso a cuidado intensivo siendo la pre-eclampsia grave la causa principal de ingreso a UCI y de muerte perinatal.

La sobrevivencia materna de $100 \%$ y la sobrevivencia perinatal de $92 \%$ obtenida en esta serie es un resultado asociado a la calidad del cuidado médico y al trabajo conjunto de perinatólogos, intensivistas y otros especialistas.

La información obtenida de permanencia en UCI es una fuente apropiada para el estudio de costos.

\section{Referencias}

1. Confidential enquiry into maternal and child health. Why mothers die 2000-2002. Sixth report on confidential enquiries into maternal death in the United Kingdom. London, Royal College of Obstetricians and Gynecologist, 2004, pp 1338.

2. Danel I, Berg C, Johnson CH, Atrash H. Magnitude of maternal morbidity during labor and delivery. United States, 1993 - 1997. Am J Public Health 2003; 93: 631-4.

3. Geller S, Rosenberg D, Cox SM, Brown M, Simonson L, Driscoll CA, et al. The continuum of maternal morbidity and mortality: Factors associated with severity. Am Journal Obstet Gynecol 2004; 191: 939-44.

4. Penney G, Brace V. Near miss audit in obstetrics. Curr Opin Obstet Gynecol 2007; 19 (2): 145-50.

5. Donoso E. Mortalidad Materna: Chile 2000-2004. Rev Chil Obstet Ginecol 2006; 71: 246-51.

6. Donoso E. Tras el cumplimiento del 5ºbjetivo del Milenio: Mortalidad Materna. Chile 2003. Rev Chil Obstet Ginecol 2010; 75 (1): 1-2.

7. Department of reproductive Health and Research World Health Organization. Beyond the numbers reviewing maternal death and complications to make pregnancy safer. Geneva WHO Press2004. Hallado en: http://www. searo.who.int/LinkFiles/Making_Pregnancy_Safer_Beyond_the_numbers.pdf

8. Geller SE, Cox SM, Callaghan WM, Berg CJ. Morbidity and mortality in pregnancy, laying the groundwork for safe motherhood. Women's Health Issues 2006; 16: 17688.

9. Keizer JL, Zwart J, Meerman RH, Harinck B, Feuth
HDM, van Roosmalen J. Obstetric intensive care admissions: A 12 year review in a terciary care centre. Eur J Obstet Gynecol Reprod Biol 2006; 128: 152-6.

10. Bouvier-Colle MH,Varnoux N, Groupe MOMS-B. Maternal mortality and severe morbidity in French regions: results of MOMS, a European multicenter investigation. J Gynecol Obstet Biol Reprod (Paris) 2001; (Suppl 6): S 5-9.

11. Mantel GD, Buchmann E, Rees H, Pattindon RC. Severe acute maternal morbidity: a pilot study of a definition for a near-miss. Br J Obstet Gynaecol 1998; 105: 985-90.

12. Prual A, Bouvier-Colle MH, de Bernis L, Bréart G. Severe maternal morbidity from direct obstetric causes in West Africa: incidence and case fatality rates. Bull WHO 2000; 78: 593-603.

13. Zhang WH, Alexander S, Bouvier-Colle MH, Macfarlane A. Incidence of severe pre-eclampsia, postpartum haemorrhage and sepsis as a surrogate marker for severe maternal morbidity in a European population-based study: the MOMS-B survey. BJOG 2005; 112 (1): 89-96.

14. Afessa B, Green B, Delke I, Koch K. Systemic Inflammatory Response Syndrome, Organ Failure, and Outcome in Critically III Obstetric Patients Treated in an ICU. Chest 2001; 120: 1271-7.

15. Vásquez DN, Estenssoro E, Canales HS, Reina R, Sáenz MG, Das Neves AV, et al. Clinicals Characteristics ond Outcomes of Obstetric Patients Requiring ICU Admission. Chest 2007; 131: 718-24.

16. Sibai BM, Ramadam MK, Usta I, Salama M, Mercer BM, Friedman SA. Maternal morbidity and mortality in 442 pregnancies with hemolysis, elevated liver enzymes, and low platelets (HELLP syndrome). Am J Obstet Gynecol 1993; 169: 1000-6.

17. Parra M, Rodrigo R, Barja P, Bosco C, Fernández $\mathrm{V}$, Muñoz H, et al. Screening test for preeclampsia through assessment of uteroplacental blood flow and biochemical markers of oxidative stress and endothelial dysfunction. Am J Obstet Gynecol 2005; 193: 1486-91.

18. Parra-Cordero M, Rodrigo R, Barja P, Bosco C, Rencoret G, Sepúlveda-Martínez A, et al. Prediction of early and late pre-eclampsia from maternal characteristics, uterine artery Doppler and markers of vasculogenesis during first trimester of pregnancy. Ultrasound Obstet Gynecol 2013; 41: 538-44.

19. Akolekar R, Syngelaki A, Poon L, Wright D, Nicolaides KH. Competing Risks Model in Early Screening for Preeclampsia by Biophysical and Biochemical Markers. Fetal Diagn Ther 2013; 33: 8-15.

20. Scazzocchio E, Figueras F, Crispi F, Meler E, Masoller N, Mula R, et al. Performance of a first-trimester screening of preeclampsia in a routine care low-risk setting. Am J 
Morbilidad materna grave en UCI - J. Hasbún et al

Obstet Gynecol 2013; 208: 203. e1-10.

21. Bujold E, Roberge S, Lacasse Y, Bureau M, Audibert F, Marcoux S, et al. Prevention of Preeclampsia and Intrauterine Growth Restriction With Aspirin Started in Early Pregnancy. A Meta-Analysis. Obstet Gynecol 2010; 116: 402-14.

22. Roberge S, Villa $\mathrm{P}$, Nicolaides $\mathrm{K}$, Giguère $\mathrm{Y}$, Vainio M, Bakthi A, et al. Early Administration of Low-Dose Aspirin for the Prevention of Preterm and Term Preeclampsia: A Systematic Review and Meta-Analysis. Fetal Diagn Ther 2012; 31: 141-6.

23. Roberge $S$, Giguère $Y$, Villa $P$, Nicolaides $K$, Vainio $M$, Forest JC, et al. Early Administration of Low-Dose Aspirin for the Prevention of Severe and Mild Preeclampsia: A Systematic Review and Meta-Analysis. Am J Perinatol 2012; 29: 551-6.

24. Roberge S, Nicolaides KH, Demers S, Villa P, Bujold E. Prevention of perinatal death and adverse perinatal outcome using low-dose aspirin: a meta-analysis. U1trasound Obstet Gynecol 2013; 41: 491-9.

25. Cantwell R, Clutton-Brock T, Cooper G, Dawson A, Dife J, Garrod D, et al. Saving Mothers' Lives: reviewing maternal death to make motherhood safer: 2006-2008. The Eighth Report of the Confidential Enquires into Medical Death in the United Kingdom. BJOG 2011; 118 (supp 1): 1-203.

26. Dellinger RP, Levy MM, Carlet JM, Bion J, Parker MM, Jaeschke R, et al. Surviving Sepsis Campaign: international guidelines for management of severe sepsis and septic shock . Crit Care Med 2008; 36 (1): 296-327.
27. Sumigama S, Ikakura A, Ota T, Okada M, Kotany T, Hayakasawa $\mathrm{H}$, et al. Placenta previa increta/percreta in Japan: a retrospective study of ultrasound findings, management and clinical course. J Obstet Gynaecol Res 2007; 33 (5): 606-11.

28. Hasbún J, Palaveccini R, Osorio R, Castro M, Carrreño P, Valdevenito R. Oclusión Temporal de arterias ilíacas internas en cesárea-histerectomía por placenta acreta: enfoque multidisciplinario. Rev Chil Obstet Ginecol 2012; 77 (1): 58-63.

29. Dias De Souza JP, Duarte G, Basile-Filhp A. Near-miss maternalmortality in developing countries. European Journal of Obstetrics \&Gynecology and Reproductive Biology 2002; 104 (1): 80.

30. Vigil-De Gracia P. Maternal deaths due to eclampsia and HELLP syndrome. Int J Gynaecol Obstet 2009; 104 (2): 90-4.

31. Gul A, Cebeci A, Aslan H, Polat I, Ozdemir A, Ceylan Y. Perinatal outcomes in severe preeclampsia-eclampsia with and without HELLP syndrome. Gynecol Obstet Invest 2005; 59 (2): 113-8.

32. Sibai BM. Diagnosis, controversies, and management of the syndrome of hemolysis, elevated liver enzymes, and low platelet count. Obstet Gynecol 2004; 103: 981-91.

33. Abramovici D, Friedman SA, Mercer BM, Audibert F, Kao L, Sibai BM. Neonatal outcome in severe preeclampsia at 24 to 36 weeks' gestation: does the HELLP (hemolysis, elevated liver enzymes, and low platelet count) syndrome matter? Am J Obstet Gynecol 1999; 180: 221-5. 